\title{
Vitamina D e Cálcio na prevenção da osteoporose
}

Exm. ${ }^{\circ}$ Senhor Director da Revista Portuguesa de Clínica Geral,

ARPCG publicou no número de Maio/Junho de 2010, um artigo de melhoria contínua da qualidade intitulado «Estudo P.O.I.S. (prevenção da osteoporose nos idosos»). ${ }^{1}$ Embora os autores mencionem o objectivo de «avaliar e garantir a qualidade», parto do princípio que pretendem descrever a experiência de um ciclo de melhoria contínua de qualidade na prescrição de cálcio e vitamina $D$ ao idoso para a prevenção da osteoporose. Comefeito, em clínica não é correcto falar de "garantia de qualidade» mas antes de «melhoria da qualidade», dado que a «garantia» muito dificilmente se pode fazer. A referida avaliação de qualidade parte do princípio que a prescrição de cálcio e vitamina $D$ previnem a osteoporose primária do idoso e, assim, as fracturas do colo do fémur e da coluna. Invocam como justificação para este procedimento, a generalizar nos idosos, ofacto de as fracturas do idoso serem frequentes e com elevada morbi-mortalidade e uma circular da DGS onde não é demonstrado o que preconiza, fundamentando-se mais na opinião que na evidência.

De momento o que se sabe é:

1. A vitamina Dé necessária para a formação do osso.

2. As fracturas da coluna e do colo do fémur são graves e relativamente frequentes nos idosos.

Contudo, não existe evidência de que a suplementação de vitamina D e ou cálcio reduza a incidência de fracturas, excepto na osteoporose secundária. ${ }^{3}$ Uma revisão sobre o assunto, que incluiu 35 ensaios com 84.585 participantes, publicada na «The Cochrane Collaboration», conclui que a vitamina D isolada não é efectiva na prevenção de fracturas da anca, da coluna ou em qualquer outra localização. ${ }^{4}$ A associação de vitamina De cálcio também não reduz a incidência de fracturas na população idosa, excepto na institucionalizada em que parece reduzir a incidência de fractura da anca. A suplementação de vitamina D com ou sem cálcio aumenta significativamente a calcémia que, por sua vez, se associa ao aumento moderado de sintomas gastrointestinais e um pequeno aumento, embora significati$v o$, de doença renal (odds ratio de 1:16, com intervalo de confiança de $95 \%$ de 1,02 e 1,33).
O procedimento preconizado no artigo em questão, para o universo da população idosa, não só não está demonstrada a sua efectividade na prevenção das fracturas como até pode ser prejudicial. Para além disso, não tem em consideração outras medidas para a prevenção de quedas e consequentes fracturas para as quais existe evidência de efectividade como: correcção da visão, redução gradual do uso de psicofármacos, implantação de pacemaker sempre que indicado, promoção de actividade física e a melhoria das condições habitacionais e ambientais. ${ }^{5}$

Em Medicina o primum non nocereé um princípio inultrapassável mas, em Medicina Preventiva, adiciona-se o princípio de «utilidade demonstrada», i.e, o efeito placebo não tem aqui aplicação.

Conclusão:pelo menos atéque esteja demonstrada a efectividade da suplementação de cálcio e de vitamina D para a prevenção primária de fracturas, tal procedimento executado de modo universal não deve ser considerado um critério de qualidade dos cuidados clínicos prestados por qualquer instituição.

Com os cumprimentos

José Mendes Nunes

Assistente Graduado Sénior da USCP de Almeirim (ACES da Lezíria)

Assistente convidado do Departamento de MGF, da Faculdade de Ciências Médicas de Lisboa, Universidade Nova de Lisboa

\section{REFERÊNCIAS BIBLIOGRÁFICAS}

1. Nunes A, Loureiro O, Donat T, Skorobohach V. Estudo P.O.I.S. (prevenção da osteoporose nos idosos). Rev Port Clin Geral 2010 Mai-Jun; 26 (3): 248-54.

2. DGS. Orientação técnica sobre suplementação de cálcio e vitamina $D$ em pessoas idosas. Circular Informativa n 13/DSCS/DSQC de 01/04/2008.

3. Homik J, Suarez-Almazor ME, Shea B, Cranney A, Well GA, Tugwell P. Calcium and Vitamin D for corticoisteroid-induced osteoporosis. Cochrane Database Syst Rev 2000; (2): CD000952.

4. Avenell A, Gillespie WJ, Gillespie LD, O'Connell D. Vitamin D and vitamin $D$ analogues for preventing fractures associated with involutional and post-menopausal osteoporosis. Cochrane Database Syst Rev 2009; (2): CD000227.

5. McClure R, Turner C, Peel N, Spinks A, Eakin E, Hughes K. Populationbased interventions for the prevention of fall-related injuries in older people. Cochrane Database Syst Rev 2005 Jan 25; (1): CD004441. 